\title{
COMPORTAMENTO DE PROGÊNIES ORIUNDAS DE RAÇAS PRIMITIVAS DE ALGODÃO HERBÁCEO FRENTE AO ATAQUE DO BICUDO ${ }^{1}$
}

\author{
FRANCISCO JOSÉ CORREIA FARIAS², MAURICE JAMES LUKEFAHR ${ }^{3}$, \\ JOAQUIM NUNES DA COSTA ${ }^{2}$ e ELÊUSIO CURVELO FREIRE ${ }^{4}$
}

\begin{abstract}
RESUMO - Com o objetivo de obter linhagens resistentes ao bicudo-do-algodoeiro (Anthonomus grandis Boheman), a Embrapa-Centro Nacional de Pesquisa de Algodão vem testando progênies oriundas de raças primitivas de algodoeiro herbáceo (Gossypium hirsutum L.) originárias do México e da América Central, que apresentam níveis aceitáveis de resistência ao bicudo. Em 1991 e 1992, as progênies em $\mathrm{BC}_{1} \mathrm{~F}_{5}$ e $\mathrm{BC}_{1} \mathrm{~F}_{6}$ oriundas das linhagens Texas 277, Texas 326 e Texas 1180, Texas 297, Texas 339, Texas 766 e Texas 1134, foram avaliadas com relação à resistência ao bicudo. $\mathrm{O}$ delineamento experimental utilizado foi o de blocos ao acaso, com seis repetições. A unidade experimental foi constituída por duas fileiras de $5 \mathrm{~m}$, sob um espaçamento de $0,75 \mathrm{~m} \mathrm{x} 0,20 \mathrm{~m}$. As parcelas foram infestadas com adultos do bicudo recém-emergidos, a uma taxa de 10.000 adultos/ha. Aos seis dias após a liberação dos adultos, as parcelas foram pulverizadas com Cipermethrim, sendo realizadas em intervalos semanais. Foram procedidas cinco avaliações através da coleta de 33 botões florais ao acaso, por parcela. Os maiores níveis de resistência ao bicudo foram obtidos pelas progênies Texas 326-95-1, Texas 277-87-5, Texas 1180-99-2, Texas 297 e Texas 339, com redução de ataque de 44,0, 41,2, 32,0, 40,4 e 36,4\%, respectivamente, em relação à testemunha CNPA 6H.
\end{abstract}

Termos para indexação: resistência do hospedeiro, melhoramento, Gossypium.

\section{BEHAVIOR OF LINES FROM COTTON PRIMITIVE RACE STOCKS TO ATTACK OF THE BOLL WEEVIL}

\begin{abstract}
The goal of the boll weevil (Anthonomus grandis Boheman) resistance program of Embrapa-Centro Nacional de Pesquisa de Algodão is to obtain cultivars that give $80 \%$ suppression when compared to commercial cultivars. The primitive cottons (Gossypium hirsutum L.) from Mexico and Central America have been known to show measurable levels of resistance to the boll weevil. In 1991 and 1992, the lines in $\mathrm{BC}_{1} \mathrm{~F}_{5}$ and $\mathrm{BC}_{1} \mathrm{~F}_{6}$ from Texas 277, Texas 326, Texas 1180, Texas 297, Texas 339, Texas 766 and Texas 1134 were evaluated to boll weevil resistance. The experiment was carried out at Campina Grande, PB, Brazil. The trial utilized was RCB with six replications. The plots were infested with newly emerged adults at the rate of 10,000 adults/ha. Six days after adults were released, the plots were sprayed with Cipermethrim. Infestation counts started three weeks after the Cipermethrim application and were made at weekly intervals. Five infestation counts were conducted by collecting 33 squares at random from each plot. The highest levels of resistance occurred in Texas 326-95-1, Texas 277-87-5, Texas 1180-99-2, Texas 297 and Texas 339 with 44.0, 41.2, 32.0, 40.4 and $36.4 \%$ of attack reduction, respectively, in relation to control CNPA $6 \mathrm{H}$.
\end{abstract}

Index terms: host resistance, breeding, Gossypium.

${ }^{1}$ Aceito para publicação em 24 de fevereiro de 1999.

${ }^{2}$ Eng. Agr., M.Sc., Embrapa-Centro Nacional de Pesquisa de Algodão (CNPA), Caixa Postal 174, CEP 58107-720 Campina Grande, PB. E-mail: farias@cnpa.embrapa.br

${ }^{3}$ Eng. Agr., Ph.D, Embrapa-CNPA.

${ }^{4}$ Eng. Agr., Dr., Embrapa-CNPA. E-mail: eleusio@cnpa.embrapa.br

\section{INTRODUÇÃO}

O bicudo-do-algodoeiro (Anthonomus grandis Boheman) é a praga de maior nocividade econômica já detectada no continente americano. Os danos causados por ele nos órgãos reprodutivos desta planta podem causar perdas de até $100 \%$, caso não haja nenhum tipo de controle (Barbosa et al., 1983). 
Uma estratégia que vem sendo utilizada com sucesso no Brasil e nos Estados Unidos na obtenção de plantas resistentes ao bicudo é a utilização de raças primitivas de algodão, oriundas do México e América Central, onde teve origem a espécie Gossypium hirsutum.

Buford et al. (1967), trabalhando em condições de laboratório, obtiveram reduções de 30 a $40 \%$ na oviposição do bicudo em diferentes tipos de algodões primitivos.

Earnheart Júnior, citado por Jones et al. (1986), identificou 49 raças silvestres de Gossypium hirsutum com níveis significativos de resistência ao bicudo, com base em testes-padrão de oviposição, em laboratório. A maioria dos materiais resistentes foi coletada, originalmente, ao longo da costa dos Estados de Guerrero, Oaxaca e Vera Cruz, no México. A resistência atribuída a este fator é do tipo antibiose/ não-preferência (Jones et al., 1986).

Em condições de campo, McCarty Junior et al. (1977) testaram linhagens em $\mathrm{BC}_{2} \mathrm{~F}_{2}$ e $\mathrm{BC}_{2} \mathrm{~F}_{3}$ oriundas de raças primitivas com fatores de resistência ao bicudo. Foram encontradas diferenças significativas em alguns anos na postura pelo bicudo nos diferentes materiais, apesar de as diferenças não excederem a 5\%. McCarty Junior et al. (1987) observaram que dois acessos de materiais primitivos convertidos para dias neutros, Texas 326DN e Texas 1180DN, os quais foram retrocruzados duas vezes com seus respectivos pais, obtiveram significativamente menos orifícios de oviposição que a testemunha Stoneville 213, em condições de laboratório.

Segundo McCarty Junior \& Jones (1989), as linhagens Texas 109DN, Texas 277-2-6DN, Texas 330DN, Texas 759DN, Texas 763DN e Texas 790DN tiveram significativamente menos bicudos em oviposição e menos maçãs atacadas que as testemunhas susceptíveis Stoneville 213 e Deltapine 41, em ensaios de campo e de laboratório. De acordo com os autores, os níveis de resistência desses materiais se aproximaram dos observados na testemunha resistente Frego Nectariless LA $81-560 \mathrm{FN}$.

No Brasil, após o aparecimento do bicudo, em 1983, a Embrapa-Centro Nacional de Pesquisa de Algodão deu início a um programa de melhoramento visando à obtenção de materiais resistentes a esta praga, utilizando essas raças primitivas de algodoeiro herbáceo. Como resultado do trabalho, em 1985 foram identificadas três fontes de resistência: Texas 1180, Texas 326-1 e Texas 277-6, as quais obtiveram, em testes de campo, significativas reduções na oviposição, quando comparadas com a testemunha CNPA 3H (Lukefahr \& Vieira, 1986). O alto nível de resistência de campo encontrado na linhagem Texas 277-2-6 foi, posteriormente, confirmado por McCarty Junior \& Jones (1989) e Bates et al. (1991).

Farias \& Lukefahr (1992) observaram, em testes de campo, que as progênies Texas 297, Texas 323 e Texas 339 obtiveram elevados níveis de resistência ao bicudo, com reduções de ataque de $62 \%, 53 \%$ e $49 \%$, respectivamente, em relação à testemunha Deltapine 16.

Em avaliações realizadas no campo, Soares \& Lara (1993) verificaram que a progênie Texas 277-2-6 evidenciou resistência do tipo não-preferência para oviposição e alimentação, com indícios de antibiose.

Carvalho et al. (1996) verificaram, em ensaios de campo, que as progênies Texas 277-8 e Texas 277-11 apresentaram elevados níveis de resistência ao bicudo, com reduções de ataque de 43,7 e $37 \%$, respectivamente.

O presente trabalho teve como objetivo avaliar a resistência ao bicudo em novas progênies de algodoeiro herbáceo oriundas das raças silvestres Texas 1180, Texas 326, Texas 277-2-6, Texas 339, Texas 766 e Texas 1134.

\section{MATERIAL E MÉTODOS}

Os experimentos foram conduzidos na área experimental da base física da Embrapa-Centro Nacional de Pesquisa de Algodão, em Campina Grande, PB, durante o período de 1991 a 1992.

As progênies utilizadas nos experimentos são oriundas de seis fontes de resistência ao bicudo, denominadas: Texas 1180, Texas 326, Texas 277-2-6, Texas 339, Texas 766 e Texas 1134, provenientes do programa de melhoramento desenvolvido pela Texas A\&M University que foram retrocruzadas com a Deltapine 16 (DPL 16), obtendo-se os $\mathrm{BC}_{1} \mathrm{~F}_{5}$.

Em 1991, foram avaliadas as progênies $\mathrm{BC}_{1} \mathrm{~F}_{5}$ oriundas das linhagens Texas 277, Texas 326 e Texas 1180, com relação à resistência ao bicudo. $\mathrm{O}$ delineamento experi- 
mental utilizado foi o de blocos ao acaso, com seis repetições. A parcela experimental constituiu-se de uma fileira de $4 \mathrm{~m}$, com espaçamento de $0,75 \mathrm{~m}$ x $0,20 \mathrm{~m}$, infestadas com adultos recém-emergidos, a uma taxa de 10.000 adultos/ha. Realizaram-se as avaliações utilizando as populações nativas de bicudo. Aos seis dias após a liberação dos adultos, foram pulverizadas as parcelas com Cypermethrin $30 \mathrm{ED}$ (40 g/ha de i.a.) com pulverizador Electrodyn. As avaliações tiveram início três semanas após a aplicação do Cypermethrin, e foram realizadas em intervalos semanais. Foram feitas cinco avaliações, com 33 botões florais coletados ao acaso. Os botões florais com orifícios de oviposição e alimentação foram colocados em recipientes de plástico separados; em seguida, determinaram-se as porcentagens de infestação, redução e orifícios de oviposição.

Em 1992, foram testadas, no campo, as novas linhagens oriundas de $\mathrm{BC}_{1} \mathrm{~F}_{5}$ ou $\mathrm{BC}_{1} \mathrm{~F}_{6}$ de Texas 297, Texas 339, Texas 766, Texas 1134 e Texas 326, com Deltapine 16. O delineamento experimental utilizado e a metodologia empregada foram semelhantes aos do experimento conduzido em 1991.

Para as análises estatísticas utilizou-se o programa SAS (1985), com o emprego dos modelos lineares gerais (PROC GLM). Na comparação das médias, utilizou-se o teste de comparação múltipla da Diferença Mínima Significativa (DMS) a $5 \%$ de probabilidade. Com vistas à homogeneidade das variâncias dentro dos tratamentos, os dados originais foram transformados para arc sen $\sqrt{x / 100}$.

\section{RESULTADOS E DISCUSSÃO}

Nas Tabelas 1, 2 e 3 encontram-se os percentuais de infestação, redução e de orifícios de oviposição das progênies oriundas das raças silvestres Texas 277, Texas 326 e Texas 1180, respectivamente. Verificaram-se diferenças significativas pelo teste de Diferença Mínima Significativa (DMS) a 5\% de probabilidade entre as progênies avaliadas. A precisão experimental verificada pelo coeficiente de variação (CV) ficou entre $23,16 \%$ e 30,99\%, valores considerados aceitáveis em avaliações dessa natureza (Lukefahr et al.,1991; Soares,1992).

Com relação às progênies oriundas da raça silvestre Texas 277, observa-se, na Tabela 1, que os materiais mais resistentes foram: Texas 277-87-5, Texas 277-89-4 e Texas 277-89-3, com níveis de redução de $41 \%$, $33 \%$ e $32 \%$, respectivamente; Texas 277-89-3 obteve o menor percentual de orifícios de oviposição. Tais resultados confirmam o alto nível de resistência dos materiais oriundos da raça Texas 277, obtidos por Lukefahr \& Vieira (1986) e verificados posteriormente por McCarty Junior \& Jones (1989), Soares \& Lara (1993) e Carvalho et al. (1996).

Quanto às progênies derivadas da Texas 326, verifica-se, na Tabela 2, que as progênies Texas 326-95-1, Texas 326-94-7 e Texas 326-93-7 obtiveram redução de ataque de 44,36 e $31 \%$, respectivamente,

TABELA 1. Avaliação da resistência ao bicudo em progênies oriundas da linhagem Texas 277. Dados de cinco coletas. Campina Grande, PB, $1991^{1}$.

\begin{tabular}{|c|c|c|c|c|c|}
\hline \multirow[t]{2}{*}{ Progênie } & \multicolumn{5}{|c|}{ № de orifícios de oviposição } \\
\hline & $\begin{array}{l}\text { Porcentagem } \\
\text { média de } \\
\text { infestação } \\
\end{array}$ & $\begin{array}{l}\text { Redução } \\
(\%)\end{array}$ & $\begin{array}{c}\text { Porcentagem de } \\
\text { orifício de oviposição }\end{array}$ & $\begin{array}{l}\text { Porcentagem } \\
\text { de oviposição } \\
\text { em } 500 \text { botões }\end{array}$ & $\begin{array}{l}\text { Redução } \\
(\%)\end{array}$ \\
\hline CNPA $6 \mathrm{H}^{2}$ & $39,7 \mathrm{a}$ & - & $25 a$ & 128 & - \\
\hline Texas $277^{3}$ & $27,1 \mathrm{~b}$ & 32 & $19 \mathrm{ab}$ & 97 & 24 \\
\hline Texas $277-87-5$ & $23,3 b$ & 41 & $16 b$ & 81 & 37 \\
\hline Texas 277-89-4 & $26,4 \mathrm{~b}$ & 33 & $18 \mathrm{ab}$ & 93 & 27 \\
\hline Texas $277-89-3$ & $27,1 b$ & 32 & $15 b$ & 80 & 37 \\
\hline Texas 277-90-3 & $30,6 a b$ & 23 & $17 b$ & 86 & 33 \\
\hline Texas $277-88-6$ & $30,2 \mathrm{ab}$ & 24 & $17 \mathrm{~b}$ & 88 & 31 \\
\hline C.V. $(\%)$ & 25,35 & & 30,31 & - & - \\
\hline DMS $(5 \%)$ & 9,67 & & 7,31 & - & - \\
\hline
\end{tabular}


TABELA 2. Avaliação da resistência ao bicudo em progênies $\mathrm{BC}_{1} \mathrm{~F}_{5}$ oriundas da raça primitiva T-326. Dados de cinco coletas. Campina Grande, PB, 1991.

\begin{tabular}{|c|c|c|c|c|}
\hline Progênie & $\begin{array}{c}\text { Média de botões } \\
\text { atacados durante o } \\
\text { ciclo }(\%)\end{array}$ & $\begin{array}{c}\text { Redução } \\
(\%)\end{array}$ & $\begin{array}{l}\text { Botões com orifícios } \\
\text { de oviposição }(\%)\end{array}$ & $\begin{array}{l}\text { № de orifícios de oviposição em } \\
500 \text { botões }\end{array}$ \\
\hline CNPA $6 \mathrm{H}^{2}$ & $49,9 \mathrm{a}$ & - & $25,8 \mathrm{a}$ & 129 \\
\hline Texas 326-95-1 & $27,8 \mathrm{~b}$ & 44 & $19,2 \mathrm{~b}$ & 96 \\
\hline Texas 326-94-7 & $31,9 b$ & 36 & $17,5 b$ & 86 \\
\hline Texas 326-93-7 & $34,4 \mathrm{~b}$ & 31 & $17,5 b$ & 86 \\
\hline Texas 326-93-4 & $35,5 b$ & 29 & $23,0 \mathrm{a}$ & 115 \\
\hline Texas 326-94-6 & $35,3 \mathrm{~b}$ & 29 & $20,9 \mathrm{ab}$ & 105 \\
\hline C.V. (\%) & 26,01 & & 23,16 & \\
\hline DMS (5\%) & 12,29 & & 6,30 & \\
\hline
\end{tabular}

TABELA 3. Avaliação da resistência ao bicudo em progênies $\mathrm{BC}_{1} \mathrm{~F}_{5}$ oriundas da raça primitiva Texas-1180. Dados de seis coletas. Campina Grande, PB, 1991.

\begin{tabular}{|c|c|c|c|c|}
\hline Progênie & $\begin{array}{c}\text { Média de botões atacados } \\
\text { durante o ciclo }(\%)\end{array}$ & $\begin{array}{c}\text { Redução } \\
(\%)\end{array}$ & $\begin{array}{l}\text { Botões com orifícios de } \\
\text { oviposição }(\%)\end{array}$ & $\begin{array}{c}\text { № de orifícios de oviposição } \\
\text { em } 500 \text { botões }\end{array}$ \\
\hline CNPA $6 \mathrm{H}^{2}$ & $42,9 a$ & - & $22,0 \mathrm{a}$ & 132 \\
\hline Texas $1180-87-2$ & $33,0 a b$ & 23 & $22,1 \mathrm{a}$ & 133 \\
\hline Texas 1180-99-2 & $29,1 b$ & 32 & $14,5 b$ & 87 \\
\hline Texas 1180-96-4 & $31,5 \mathrm{ab}$ & 27 & $21,3 \mathrm{a}$ & 128 \\
\hline Texas 1180-97-1 & $31,9 \mathrm{ab}$ & 26 & $19,4 a b$ & 116 \\
\hline Texas 1180-95-6 & $33,1 \mathrm{ab}$ & 23 & $16,4 \mathrm{ab}$ & 98 \\
\hline C.V. (\%) & 30,99 & & 29,61 & \\
\hline DMS (5\%) & 12,37 & & 6,79 & \\
\hline
\end{tabular}

e que as progênies Texas 326-94-7 e Texas 326-93-7 apresentaram apenas $17,5 \%$ dos botões com orifícios de oviposição. Esses resultados estão de acordo com os obtidos por Lukefahr et al. (1991), cuja progênie, oriunda da Texas 326 obteve redução de $47,9 \%$ de botões infestados em relação à testemunha susceptível Deltapine 16, enquanto Carvalho et al. (1996) verificaram, na progênie 326-1, redução de ataque de apenas $24,1 \%$ em relação à testemunha CNPA 2H. Considerando-se que a resistência é relativa, tal discordância deveu-se, principalmente, aos diferentes graus de ataque do bicudo nas testemunhas utilizadas.

Na Tabela 3 encontram-se os dados referentes às progênies oriundas da Texas 1180, na qual se verifi- ca que o teste foi significativo somente na progênie Texas 1180-99-2, com redução de infestação de 32\%, sendo que na progênie Texas 1180-99-2, 14,5\% dos botões apresentaram orifícios de oviposição; tais resultados estão de acordo com os obtidos por Lukefahr \& Vieira (1986), cujos materiais oriundos da Texas 1180 obtiveram elevados níveis de resistência ao bicudo. Esse elevado grau de resistência foi posteriormente confirmado por Bates et al. (1991), Soares \& Lara (1993) e Carvalho et al. (1996).

Com relação às novas fontes de resistência obtidas dos cruzamentos de materiais primitivos da Coleção do Texas com a cultivar Deltapine 16, foram testados em 1992 cinco novos materiais, cujos resultados se encontram na Tabela 4. Verifica-se que o 
TABELA 4. Níveis de infestação em novas linhagens selecionadas de G. hirsutum para resistência ao bicudo. Campina Grande, PB, 1992.

\begin{tabular}{|c|c|c|c|c|c|c|c|c|}
\hline \multirow[t]{2}{*}{ Linhagem } & \multicolumn{6}{|c|}{ Coleta de botões } & \multirow{2}{*}{$\begin{array}{l}\text { Média de } \\
\text { infestação }\end{array}$} & \multirow{2}{*}{$\begin{array}{l}\text { Porcentagem } \\
\text { de redução }\end{array}$} \\
\hline & $1^{\mathrm{a}}$ & $2^{-\mathrm{a}}$ & $3^{\mathrm{a}}$ & $4^{\mathrm{a}}$ & $5^{-\mathrm{a}}$ & $6^{-\mathrm{a}}$ & & \\
\hline CNPA- 6H (Test.) & 10,4 & 42,0 & 34,0 & 19,0 & 15,0 & 74,0 & $32,4 a$ & - \\
\hline Texas $297 x$ DPL-16 $\left(\mathrm{F}_{6}\right)$ & 6,6 & 29,0 & 12,6 & 9,6 & 10,0 & 48,0 & $19,3 b$ & 40,4 \\
\hline Texas 339xDPL-16 $\left(\mathrm{F}_{6}\right)$ & 9,4 & 20,0 & 11,6 & 11,4 & 13,2 & 58,0 & $20,6 b$ & 36,4 \\
\hline Texas 766xDPL-16 $\left(\mathrm{F}_{5}\right)$ & 16,0 & 36,0 & 23,6 & 15,0 & 14,4 & 61,0 & $27,7 \mathrm{a}$ & 14,5 \\
\hline Texas $1134 x$ DPL-16 $\left(\mathrm{F}_{6}\right)$ & 17,6 & 37,0 & 22,4 & 17,4 & 16,6 & 58,4 & $28,2 \mathrm{a}$ & 13,0 \\
\hline Texas 326xDPL-16 $\left(\mathrm{F}_{6}\right)$ & 16,0 & 40,0 & 18,4 & 17,4 & 17,4 & 63,4 & $28,8 \mathrm{a}$ & 11,7 \\
\hline DMS & - & - & - & - & - & - & 6,98 & - \\
\hline C.V. $(\%)$ & - & - & - & - & - & - & 18,79 & - \\
\hline
\end{tabular}

${ }^{1}$ Dados transformados em arc sen; nas colunas, médias seguidas de letras iguais não diferem entre si a $5 \%$ de probabilidade pelo teste da Diferença Mínima Significativa (DMS).

coeficiente de variação obtido foi de 18,79\%, o que indica uma aceitável precisão experimental. Observa-se, ainda, que houve diferenças significativas entre as progênies testadas por meio do teste de DMS, a $5 \%$ de probabilidade. Constata-se que os maiores níveis de resistência foram obtidos pelas progênies híbridas Texas 297 e Texas 339, com redução de ataque de 40,4 e 36,4\%, respectivamente. Estes níveis de resistência são semelhantes aos obtidos por Lukefahr et al. (1991), em trabalhos conduzidos no Estado do Texas. Tais resultados sugerem que a resistência dos materiais é herdável, e que pode ser transferida para cultivares com características agronômicas aceitáveis, conforme foi verificado por Carvalho et al. (1996) ao incorporarem a precocidade e características tecnológicas de fibras em progênies com elevado grau de resistência ao bicudo.

Segundo McCarty Junior \& Jones (1989), o mecanismo envolvido neste tipo de resistência é de não-preferência ou antixenose (Painter, 1951); entretanto, Jones et al. (1986) afirmam que esta resistência é do tipo não-preferência/antibiose, hipótese confirmada por Hedin \& McCarty Junior (1990) ao observarem que os botões florais das progênies oriundas das raças silvestres possuíam os menores teores de açúcares, em comparação com os materiais susceptíveis.

\section{CONCLUSÕES}

1. Os fatores de resistência presentes nos materiais silvestres são herdáveis e passíveis de serem transferidos para cultivares comerciais.
2. Os maiores níveis de resistência obtidos são apresentados pelas progênies $\mathrm{BC}_{1} \mathrm{~F}_{5}$ provenientes do retrocruzamento de Texas 277-87-5, Texas 326-95-1 e Texas 1180-99-2 com DPL 16 com redução de ataque de $41 \%, 44 \%$ e $32 \%$, respectivamente.

3. As novas linhagens $\mathrm{BC}_{1} \mathrm{~F}_{5}$ das fontes Texas 297 x DPL 16 e Texas 339 x DPL 16 apresentam altos níveis de resistência com reduções de ataque de 40,4 e $36,4 \%$, respectivamente.

\section{REFERÊNCIAS}

BARBOSA, S.; BRAGA SOBRINHO, R.; LUKEFAHR, M.J.; BEINGOLEA, G.O. Relatório sobre a ocorrência do bicudo do algodoeiro Anthonomus grandis Boheman, Boll weevil, no Brasil, e recomendações para a sua erradicação. Campina Grande: Embrapa-CNPA, 1983. 12p.

BATES, S.D.; WALKER, J.K.; SMITH, C.W. Studies of boll weevil resistance in two converted, day-neutral cotton race stocks. Southwestern Entomologist, v.16, n.4, p.317-329, 1991.

BUFORD, W.T.; JENKINS, J.N.; MAXWELL, F.G. A laboratory technique to evaluate boll weevil oviposition preference among cotton lines. Crop Science, v.7, p.579-581, 1967.

CARVALHO, L.P. de; LUKEFAHR, M.J.; FARIAS, F.J.C.; VIEIRA, R. de M.; MOREIRA, J. de A.N.; COSTA, J.N. da. Seleção de algodoeiro com resistência ao bicudo. Pesquisa Agropecuária Brasileira, Brasília, v.31, n.3, p.195-199, mar. 1996.

Pesq. agropec. bras., Brasília, v.34, n.12, p.2235-2240, dez. 1999 
FARIAS, F.J.C.; LUKEFAHR, M.J. Utilização de fontes primitivas de algodão herbáceo no programa de melhoramento visando resistência ao bicudo. Relatório Técnico Anual do Centro Nacional de Pesquisa de Algodão: 1990-1991. Campina Grande, 1992. p.318-321

HEDIN, P.A.; McCARTY JUNIOR, J.C. Possible roles of cotton bud sugars and terpenoins in oviposition by the boll weevil. Journal of Chemical Ecology, v.16, n.3, p.757-772, 1990 .

JONES, J.E.; WEAVER, J.B.; SHUSTER, M.F. Plantas resistentes ao bicudo. In: BARBOSA, S.; LUKEFAHR, M.J.; BRAGA SOBRINHO, R. (Eds.). $\mathrm{O}$ bicudo do algodoeiro. Brasília: EmbrapaDDT, 1986. p.221-249.

LUKEFAHR, M.H.; SCOTT JUNIOR, A.W.; JONES, J.E.; McCARTY JUNIOR, J.E.; SMITH, W.C. Evaluation of cotton having resistance to the cotton boll weevil. In: RIO FARMS INCORPORATION. Research report 1990-1991. Monte Alto, TX, 1991. p.38-40.

LUKEFAHR, M.H.; VIEIRA, R.M. New sources of boll weevil resistance in primitive race stocks of Gossypium hirsutum. In: BELTWIDE COTTON PRODUCTION CONFERENCE, 1986, Memphis. Proceedings. Memphis: National Cotton Council, 1986. p.493-495.
McCARTY JUNIOR, J.C.; JENKINS, J.N.; PARROT, W.L. Boll weevil resistance, agronomic caracteristics and fiber quality in progenies of a cotton cultivar crossed with 20 primitives stocks. Crop Science, v.17, p.5-7, 1977.

McCARTY JUNIOR, J.C.; JENKINS, J.N.; PARROT, W.L. Genetic resistance to boll weevil oviposition in primitive cotton. Crop Science, v.27, p.263-264, 1987.

McCARTY JUNIOR, J.C.; JONES, J.E. Boll weevil (Coleoptera: Curculionidae) Non preference for primitive cotton. Journal of Economic Entomology, v.82, n.1, p.298-300, 1989.

PAINTER, R.H. Insect resistance in crop plants. New York: MacMillan, 1951. 520p.

SAS INSTITUTE. SAS user's guide: statistics. Version 6.03, 5.ed. Cary, NC, 1985. 956p.

SOARES, J.J. Aspecto da resistência do algodoeiro (Gossypium hirsutum L.) a Anthonomus grandis Boheman, 1843 (Coleoptera: Curculionidae). Jaboticabal: UNESP-FCA, 1992. 110p. Dissertação de Mestrado.

SOARES, J.J.; LARA, F.M. Não-preferência para alimentação e oviposição de Anthonomus grandis Boheman 1843 , por diferentes genótipos de algodoeiro. Científica, v.21, n.2, p.333-338, 1993. 